\title{
Utility of glutamine synthetase immunohistochemistry in identifying features of regressed cirrhosis
}

\author{
Rouba Hadi $\mathbb{1}^{1} \cdot$ Kseniya Shin $^{1} \cdot$ Nicholas Reder $^{1} \cdot$ Lindsay Alpert $^{2} \cdot$ Lisa Koch $^{1} \cdot$ Won-Tak Choi ${ }^{3} \cdot$ Paul E. Swanson ${ }^{1}$. \\ John Hart ${ }^{2} \cdot$ Maria Westerhoff ${ }^{4}$
}

Received: 7 June 2019 / Accepted: 13 July 2019 / Published online: 7 August 2019

(c) United States \& Canadian Academy of Pathology 2019

\begin{abstract}
The prevailing view that cirrhosis is irreversible has been challenged. It has been proposed that varying degrees of fibrosis regression can be achieved if the injurious agent is removed. In the normal liver, glutamine synthetase immunostaining is present around central veins. In regressed cirrhosis, although fibrous bands between portal tracts and central veins may largely be resorbed, the abnormal portal tract-central vein adherence often remains. Hence, we hypothesized that aberrant glutamine synthetase positivity adjacent to portal tracts would help identify regressed cirrhosis. We performed glutamine synthetase immunohistochemistry on 49 liver specimens (16 regressed cirrhosis, 18 cirrhotic, and 15 normal livers). Qualification for regressed cirrhosis required the following histologic features: curved, delicate incomplete septa, portal tract-central vein adhesions, and portal tract "remnants" (portal tracts with no venous branch). Out of 16, 14 regressed cirrhosis cases had baseline cirrhosis established based on previous biopsy or signs of cirrhosis based on physical exam, laboratory, and radiological findings. All regressed cirrhosis cases $(100 \%)$ had areas of aberrant glutamine synthetase positivity adjacent to portal tracts, indicating that portal tract-central vein approximation had occurred $(p<0.001$ compared to all other categories). No normal cases had glutamine synthetase positivity adjacent to portal tracts, and half of cirrhosis cases had areas showing features of regression, with focal glutamine synthetase positivity adjacent to portal tracts. Overall, glutamine synthetase expression showed highly significant differences among the three categories $(p<0.001)$. This study shows that aberrant glutamine synthetase positivity adjacent to portal tracts is present in regressed cirrhosis and can be useful in identifying regressed cirrhosis when it is histologically suspected.
\end{abstract}

\section{Introduction}

Liver cirrhosis affects approximately 1 million adults in the United States, and is one of the leading causes of chronic

Rouba Hadi

hadir2@uw.edu

1 Department of Anatomic Pathology, University of Washington Medical Center, 1959 NE Pacific St, NE 110, Seattle, WA 98195, USA

2 Department of Anatomic Pathology, University of Chicago, A27 S Maryland Ave, Chicago, IL 60637, USA

3 Department of Anatomic Pathology, University of California San Francisco, 505 Parnassus Avenue, Room M-552, San Francisco, CA 94143, USA

4 Department of Anatomic Pathology, University of Michigan Health System, NCRC building 352800 Plymouth Road, Ann Arbor, MI 48109, USA disease worldwide [1, 2]. Over half of all individuals with cirrhosis are unaware that they have the disease, since it can be asymptomatic until functional liver decompensation begins $[1,3]$. Symptoms of decompensation are severe, and include ascites, spontaneous bacterial peritonitis, hepatic encephalopathy, and variceal bleeding [3]. Mortality from cirrhosis is high, estimated at $>25 \%$ per 2-year interval [1]. There are several etiologies of cirrhosis, the most common of which are alcoholic liver disease, chronic viral hepatitis (hepatitis B and C), nonalcoholic fatty liver disease, and hemochromatosis [3].

Cirrhosis is defined as the presence of regenerative nodules separated by bands of fibrosis. Intervening fibrous septa distort hepatic parenchyma through the connection of portal-to-portal, portal-to-central, or central-to-central veins. The result is the isolation of islands of parenchyma with associated disruption of normal vascular architecture and function [4]. Parenchymal extinction lesions are an important feature of chronic liver disease and cirrhosis, and are 
believed to be the starting point of the latter disease process $[4,5]$. When the liver is injured and the vasculature is compromised, the area of injury becomes a parenchymal extinction lesion or an area with loss of contiguous hepatocytes, as illustrated in Fig. 1. With continuous injury, numerous parenchymal extinction lesions can develop, resulting in septal formation and, ultimately, the nodular fibrosis that in part defines cirrhosis $[4,6]$.

Historically, cirrhosis has been assumed to be an irreversible process. However, this concept of irreversibility has been challenged in recent years with studies proving

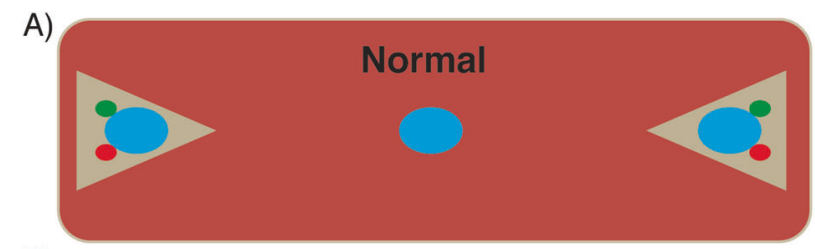

B)

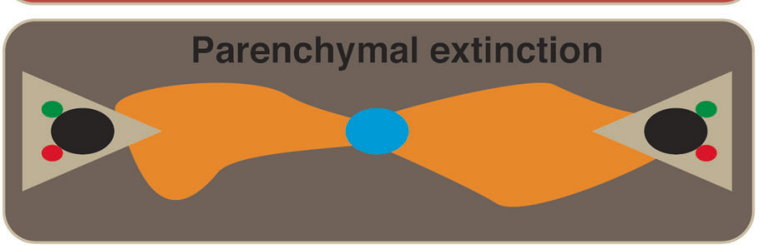

C)

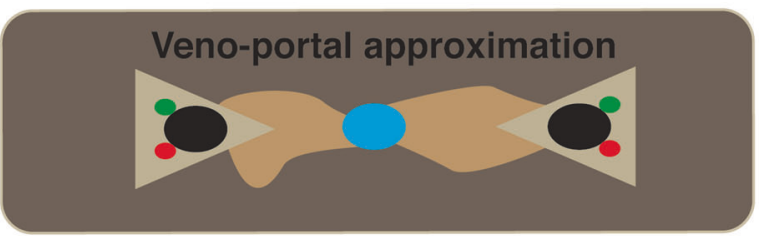

D)

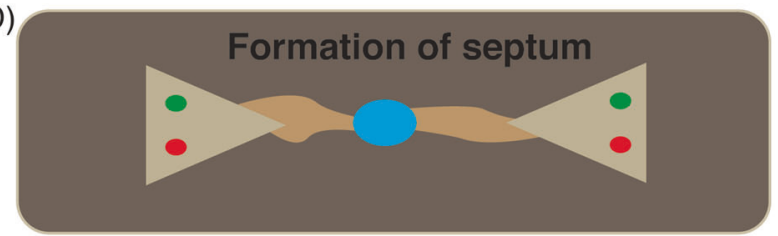

E)

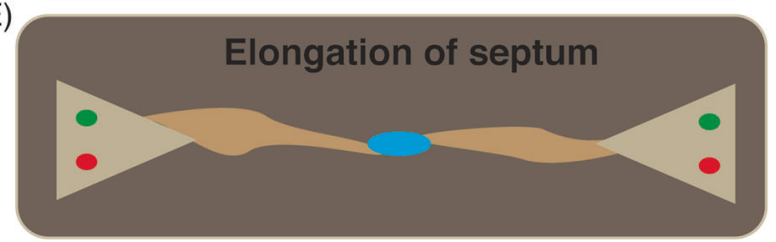

F)

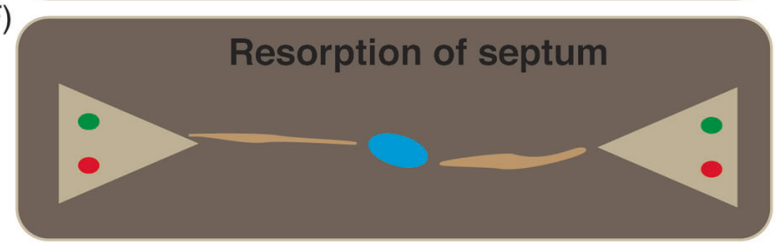

Fig. 1 Diagram depicting progression of normal liver (a) from injury to resorption/regression. Chronic injury leads to parenchymal extinction and the eventual approximation of portal tracts to central hepatic veins $(\mathbf{b}, \mathbf{c})$; there is then obliteration of portal veins and fibrous adhesion formation between the portal tract and central vein, which may expand over time (d, e); when regression occurs (f), there is resorption of fibrous septa, but the portal-central vein approximation remains; adapted with permission from Wanless et al. [6] that, with time, some advanced cases can show a significant decrease in fibrosis [6]. It has been repeatedly shown that the elimination of the causative injury or the use of effective treatment can lead to parenchymal regeneration, regression of fibrosis, and ultimately, clinical improvement [7]. As a result, some authors now suggest a change in conventional terminology and to abandon the use of the word cirrhosis entirely, as it implies an "end-stage" liver disease process [7]. In a recent study, Sun et al. [8] even proposed a change in the assessment of liver biopsies, emphasizing the significance of addressing the "dynamic changes in fibrosis." Their classification system includes categories such as predominantly "progressive fibrosis" and predominantly "regressive fibrosis". All in all, such studies point to a significant change in perspective, one that is also informed by significant advances in the treatment of liver disease, such as direct acting antivirals for hepatitis C [9].

Proposed histologic features of cirrhosis regression, include delicate incomplete fibrous septa; reduction in portal tract collagen and loss of portal veins; hepatocyte migration (hepatocytes in close proximity to hepatic arteries and bile ducts); morphologically different populations of hepatocytes in adjacent nodules; and, importantly, an approximation of central veins to portal tracts [6] (Fig. 1).

In light of advancements in the treatment of liver diseases, the histologic manifestations of regression may begin to be encountered more frequently. Pathologists should be aware of this phenomenon and of these histologic changes in order to provide clinicians with important information that could help guide appropriate management.

Identifying regressed cirrhosis, however, can be challenging. Anticipating that juxtaposition of ventral veins to portal triads is a cardinal feature of regression, and knowing that glutamine synthetase expression is characteristically limited to pericentral hepatocytes in normal livers, we hypothesized that perivenular glutamine synthetase immunoreactivity in a periportal distribution might be a marker of regression that could be applied to biopsy and excision specimens.

\section{Materials and methods}

This study was approved by the Human Subjects Research Division, Institutional Review Board (IRB), University of Washington Medical Center, (Seattle, WA, USA). Department of Pathology databases were searched for patients with the following histologic diagnosis: normal liver; features of regression; and, cirrhosis. Additional cases drawn from similar searches were also obtained from the University of California San Francisco Medical Center (San Francisco, CA, USA). Regression cases were evaluated for three main 
criteria as defined by Wanless et al. [6]: delicate incomplete fibrous septa; portal tract remnants with loss of portal veins (estimating the percentage of obliterated portal veins in approximately 10 portal tracts/section); and, veno-portal approximation. All cases of cirrhosis demonstrated wellformed parenchymal nodules surrounded by fibrous bands. We also examined whether there was histologic evidence of central vein obliteration.

Liver specimens from 49 patients were included in this study. The mean age of patients (30 males and 19 females) was 57 years. Etiologies for liver disease included hepatitis C virus $(n=19)$, hepatitis B virus $(n=2)$, cryptogenic cirrhosis $(n=4)$, nonalcoholic steatohepatitis $(n=4)$, alcoholic cirrhosis $(n=7)$, and autoimmune hepatitis $(n=$ 2). In the regressed cirrhosis group, three patients had hepatitis $\mathrm{C}$ treatment with viral eradication, one patient had hepatitis B treatment, and two alcoholic cirrhosis patients had stopped drinking (with reliable clinical documentation of prolonged sobriety). Additional clinical information obtained included the presence or absence of varices, the presence or absence of ascites, platelet count (categorized into counts less than or greater $150 \times 10^{9} / \mathrm{L}$ ), and albumin values (categorized into values less than or greater than $3.5 \mathrm{~g} / \mathrm{dL}$ ). These clinical parameters were given a score of 1 or 0 . (1: presence of varices, presence of ascites, platelets $<150 \times 10^{9} / \mathrm{L}$ and albumin $<3.5 \mathrm{~g} / \mathrm{dL}$ and clinically suspected cirrhosis; 0: absence of varices, absence of ascites, platelets $>150 \times 10^{9} / \mathrm{L}$ and albumin $>3.5 \mathrm{~g} / \mathrm{dL}$ and $n o$ clinically suspected cirrhosis). Of the 49 cases, 16 were regressed cirrhosis cases, 18 were cirrhosis cases and 15 were architecturally intact, non-inflamed liver parenchyma from resections for neoplasm, constituting our "normal" cases. There were 44 explants/resections and 5 biopsies. Regressed cirrhosis cases consisted of 4 biopsies and 12 explants/resections, while cirrhosis cases consisted of 1 biopsy and 17 explants/resections (Table 1).

Table 1 Etiologies and clinical information for cases used in the study

\begin{tabular}{|c|c|c|c|c|c|c|c|c|c|}
\hline Total $\mathrm{N}=49$ & $\begin{array}{c}\text { Obliteration } \\
\text { of Portal } \\
\text { Vein }\end{array}$ & $\begin{array}{c}\text { Obliteration } \\
\text { of Central } \\
\text { Vein }\end{array}$ & $\begin{array}{c}\text { Curved } \\
\text { Delicate } \\
\text { Incomplete } \\
\text { Septa }\end{array}$ & $\begin{array}{l}\text { GS Confirm } \\
\text { PT-CV } \\
\text { Approximati } \\
\text { on }\end{array}$ & $\begin{array}{c}\text { Clinically } \\
\text { Suspected } \\
\text { Cirrhosis }\end{array}$ & Varices & Ascites & $\begin{array}{l}\text { Platelets } \\
<150\end{array}$ & $\begin{array}{l}\text { Albumin } \\
<3.5\end{array}$ \\
\hline $\begin{array}{l}\text { Regressed } \\
\text { Cirrhosis }(\mathrm{N}=16)\end{array}$ & 16 & 0 & 16 & 16 & 14 & 5 & 5 & 11 & 4 \\
\hline Cirrhosis $(\mathrm{N}=18)$ & 15 & 8 & 9 & 10 & 17 & 8 & 11 & 14 & 11 \\
\hline Normal $(\mathrm{N}=15)$ & 0 & 0 & 0 & 0 & 0 & $\mathrm{~N} / \mathrm{A}$ & $\mathrm{N} / \mathrm{A}$ & $\mathrm{N} / \mathrm{A}$ & $\mathrm{N} / \mathrm{A}$ \\
\hline
\end{tabular}

Regressed Cirrhosis

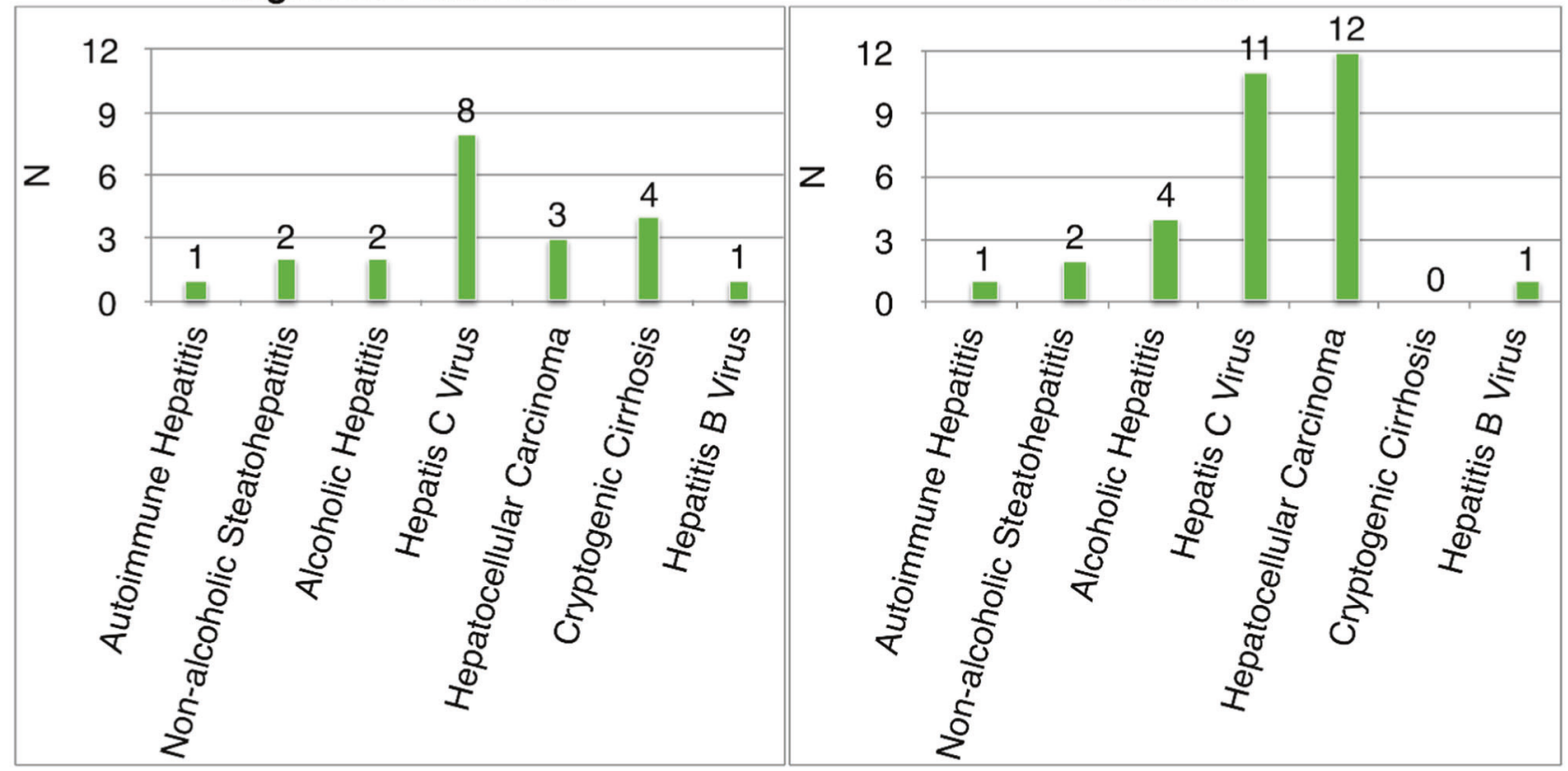

Etiologies for liver disease included Hepatitis C virus $(n=19)$, Hepatitis B virus $(n=2)$, cryptogenic cirrhosis $(n=4)$, nonalcoholic steatohepatitis $(n=4)$, alcoholic hepatitis $(n=7)$, and autoimmune hepatitis $(n=2)$. 
Slides prepared from one representative block of each case (randomly chosen if multiple blocks were available) were stained with hematoxylin-eosin and Masson's trichrome, the latter to evaluate the extent of fibrosis. An immunohistochemical assay for Glutamine synthetase (clone 6: total protein concentration of $\sim 10 \mathrm{mg} / \mathrm{ml}$; BioCare Medical, Pacheco, CA, USA) was also performed, using a polymer-based immunoperoxidase method (Leica Bond Polymer Refine Detection Kit, Leica Biosystems Inc., Buffalo Grove, IL, USA) and a hematoxylin counterstain. Immunohistochemical stains were performed on the Bond III testing platform (Leica, Buffalo Grove, IL). Cases demonstrating aberrant positive staining around portal tracts on glutamine synthetase immunohistochemistry were given a score of 1 , while those that did not show this approximation were given a score of 0 . Chi-square $\left(\chi^{2}\right)$ or Fisher's exact tests were used to determine differences in glutamine synthetase status among different histologic fibrosis categories. All statistical analyses were performed using R v3.3.1. ( $R$ Foundation for Statistical Computing, Vienna, Austria). $p$ Value $<0.001$ was considered statistically significant.

\section{Results}

All histologic features of regressed cirrhosis, including obliteration of the portal vein (up to $60 \%$ of portal veins obliterated of multiple cases examined), delicate incomplete fibrous septa and veno-portal approximation, were present in the regression cases (Fig. 2). Of the central veins recognized in regressed cirrhosis cases, there was no evidence of venous obliteration. While the majority of cirrhosis cases showed classical histologic features of cirrhosis (parenchymal nodule formation with bridging fibrosis), a number of these cases also demonstrated at least some features of regressed cirrhosis.

All cases of regressed cirrhosis had aberrant glutamine synthetase immunoreactivity around or adjacent to portal tracts, consistent with approximation of central vein to portal tract (Fig. 3a, b). No cases with normal parenchymal architecture had aberrant glutamine synthetase positivity around portal tracts; rather, all showed the expected pattern of glutamine synthetase staining around central veins (Fig. 3d). Nine (50\%) of the cirrhotic cases had focal
Fig. 2 a Hematoxylin and eosin of regressed cirrhosis case (low power view), demonstrating the absence of classical features of cirrhosis (lack of well-formed parenchymal nodules and bridging fibrosis). b, c Masson trichrome of regressed cirrhosis cases highlighting delicate, incomplete fibrous septa. d, e Hematoxylin and eosin of regressed cirrhosis cases (higher power views) demonstrating the obliteration of portal veins and veno-portal approximation, respectively
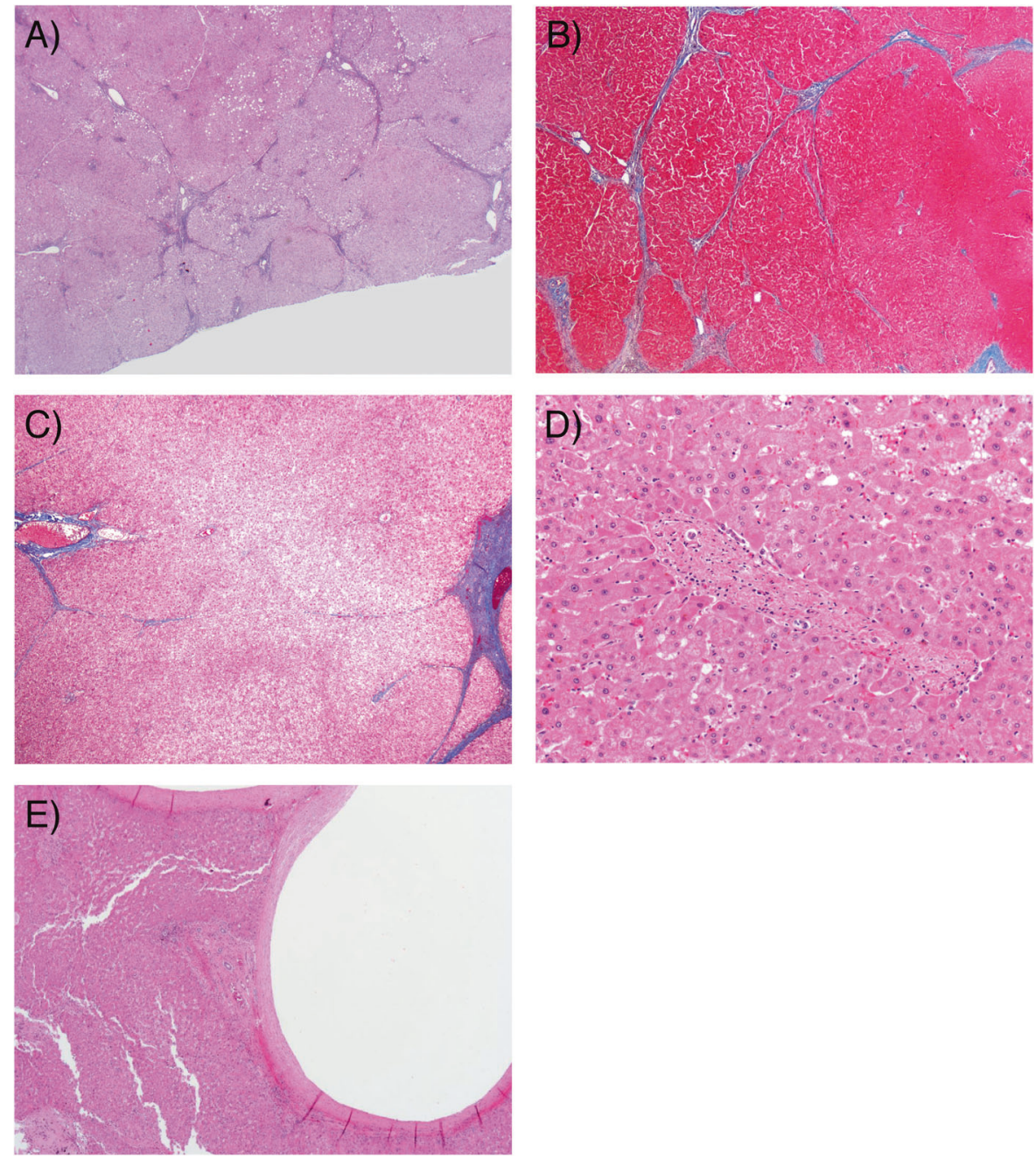
Fig. 3 a, b Glutamine synthetase immunohistochemistry of regression cases demonstrating positive glutamine synthetase staining around portal tracts. c Glutamine synthetase immunohistochemistry of a cirrhosis case with focal, aberrant glutamine synthetase staining around portal tract. d Glutamine synthetase immunohistochemistry of normal cases demonstrating expected glutamine synthetase staining around central veins, with no positive staining identified around portal tracts. e Glutamine synthetase immunohistochemistry of cirrhosis cases demonstrating complete loss of glutamine synthetase staining with f Masson trichrome highlighting nodular regeneration and fibrosis (demonstrating classic histologic features of cirrhosis)
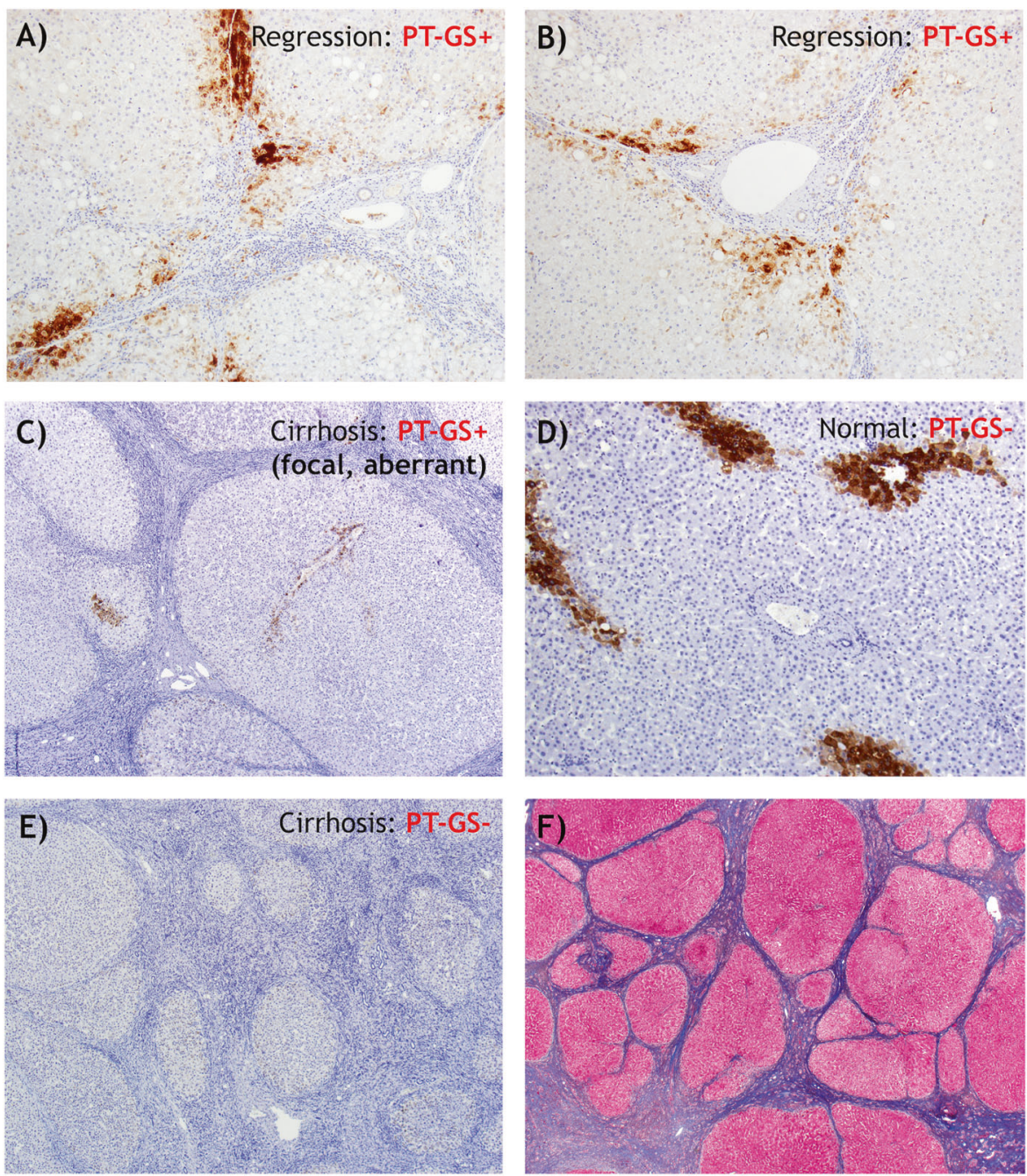

glutamine synthetase immunoreactivity around portal tracts (Fig. 3c). In seven of these nine cases, review of H\&E stains prompted by the latter observation identified areas containing histologic features of regression (most often the presence of more delicate and focally perforated fibrous septa). Remaining cirrhotic cases had almost complete loss of glutamine synthetase or only perivenular reactivity (Fig. 3e, f) (Table 2). A chi-square test showed statistically significant differences in glutamine synthetase immunoreactivity around portal tracts between regressed cirrhosis, cirrhosis, and normal livers $(p<0.0003)$.

\section{Discussion}

As proposed by Wanless et al., the progression of liver injury to cirrhosis and subsequent regression follows a reproducible sequence: ongoing liver injury leads to advanced-stage disease and cirrhosis with parenchymal extinction; the death of hepatocytes and liver parenchyma
Table 2 Summary of results of glutamine synthetase immunohistochemistry performed on all cases. Ratios depict cases with glutamine synthetase positivity around portal tracts out of total number of cases per category

\begin{tabular}{ll}
\hline$N=49$ & $\begin{array}{l}\text { Portal tract positivity } \\
\text { for Glutamine synthetase }\end{array}$ \\
\hline Regressed cirrhosis $(N=16)$ & $16 / 16 p<0.0003$ \\
Cirrhosis $(N=18)$ & $9 / 18$ (aberrant, focal) \\
Normal $(N=15)$ & $0 / 15$ \\
\hline
\end{tabular}

results in the approximation of the central veins to the portal tracts; fibrotic bands then replace dead regions of parenchyma, resulting in adhesions between the approximated portal tracts and central veins; concurrent loss of associated portal veins leaves portal tracts composed of just a hepatic artery and bile duct; eventually, hepatocytes begin to regenerate and proliferate, allowing for the resorption of thick fibrous bands and leaving behind thin and delicate incomplete fibrous septa. Although regression in cirrhosis 
involves significant parenchymal regeneration and resorption of fibrosis, the hepatic architecture does not revert to a completely normal state. Rather, because of parenchymal extinction in earlier phases of advancing fibrosis, the resorption of fibrous septa typically results in the approximation of the central vein to the portal tract $[5,8,10-12]$.

Unfortunately, the histologic features of regression may not always be easy to identify. While the lack of thick fibrosis and parenchymal nodule formation is clear, the proposed histopathologic features of regression distinct from normal may be challenging to recognize, even for an experienced pathologist. Hence, although certain aspects of a case may contain features that are suspicious for regression, a definitive diagnosis of "regressed cirrhosis" is not an easy one, especially in tissue samples of limited size. In this setting extra-morphologic clues or techniques that augment standard histomorphology, such as immunohistochemistry, may be of particular value (Fig. 4).

Glutamine synthetase is an enzyme that is normally expressed in hepatocytes that surround central veins and its expression is associated with activity of the Wnt/ $\beta$-catenin pathway. An activated Wnt pathway induces glutamine synthetase transcription factors.

Nonperivenular overexpression of glutamine synthetase in hepatocytes has been linked to focal nodular hyperplasia and neoplasms (hepatocellular carcinoma), which may be related to aberrant activation or mutation in the Wnt/ $\beta$ catenin pathway. In liver that is regnerating, $\beta$-catenin is activated in hepatocytes surrounding central veins [13, 14]. This then stimulates glutamine synthetase transcription factors and ultimately results in perivenular expression of glutamine synthetase. Studies have noted both increased glutamine synthetase expression in damaged and regenerating hepatocytes, as well as complete loss of expression in cirrhotic livers [13, 15]. A study by Fleming et al. [13] proposes the loss of endothelium-hepatocyte proximity/ contact in cirrhosis to account for loss of glutamine synthetase expression. Knowing both the normal pattern of glutamine synthetase expression and the implications of deviation from normal staining, we employed immunohistochemistry to determine if changes in the expression of glutamine synthetase might aid in the identification of what in our experience is the most often cryptic histologic feature of regression: the juxtaposition of central veins to portal triads. Since increased glutamine synthetase immunoreactivity is expected around central veins in regenerating hepatocytes, we hypothesized that when the aberrant approximation of the central vein to portal tract is present in post-cirrhotic liver, there should be significant increased glutamine synthetase staining around portal tracts. Thus, the presence of glutamine synthetase around or immediately adjacent to portal tracts, might serve as evidence of ongoing or complete regression.

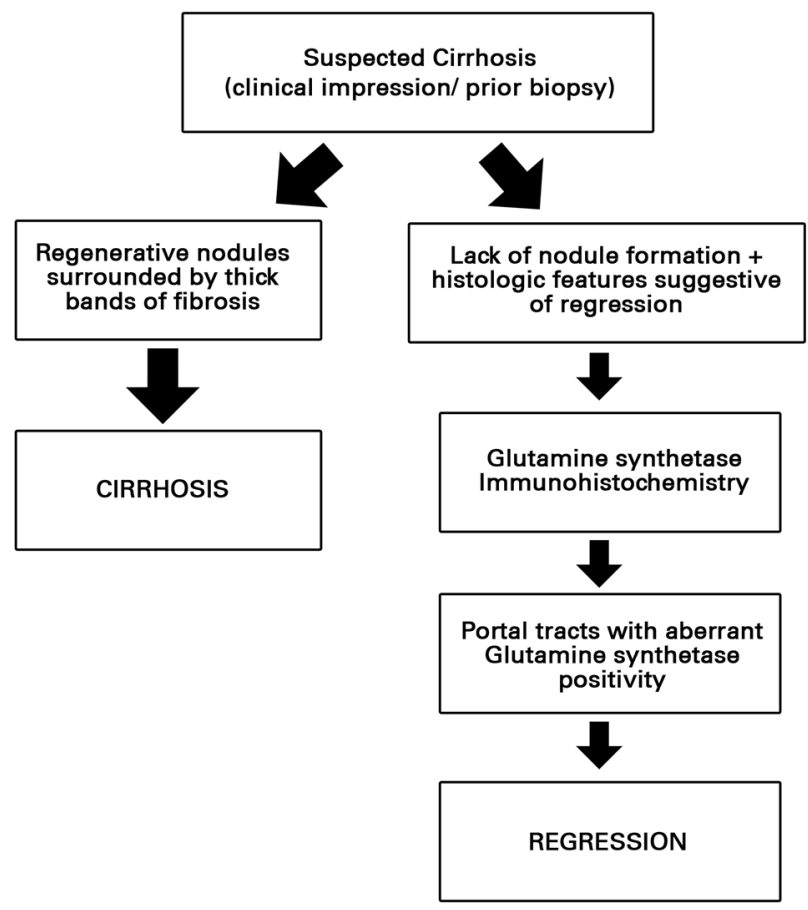

Fig. 4 Algorithm of proposed mechanism for utilization of glutamine synthetase immunohistochemistry for presumed cirrhosis cases with features suggestive of possible regression

In this study, all examples of regressed cirrhosis had the pathognomonic hematoxylin and eosin-based histologic features of this condition, including delicate incomplete fibrous septa, absent portal veins, and central veins in close proximity to portal tracts. The majority of these cases also had either a previous liver biopsy that was typical of cirrhosis, or a high clinical suspicion for advanced stage liver disease. Glutamine synthetase immunoreactivity was always present around portal tracts in regressed cirrhosis cases, supporting the potential for glutamine synthetase to be used as a marker of the approximation of central vein to portal triad in this setting. In contrast, our cases of cirrhosis (all of which demonstrated the expected histology of cirrhosis with well-formed nodules and thick fibrosis) were typically completely negative for glutamine synthetase, though nine cases exhibited faint, focal staining around portal tracts with or without aberrant reactivity scattered throughout the parenchyma. Given that regeneration in advanced fibrosis and regression appear to represent a continuum of change, this finding is not entirely unexpected. It also supports the changing perspective regarding chronic liver disease, most notably described by Quaglia et al. [7], as our emphasis shifts towards a "dynamic balance between the deposition of extracellular matrix in the setting of disease progression or extracellular matrix resorption...". Furthermore, it is important to note that varying, aberrant patterns of glutamine synthetase expression have been 
shown in advanced cirrhosis cases, particularly interface glutamine synthetase expression in association with severe cholestasis as well as hepatocyte progenitor cellassociated glutamine synthetase expression in chronic liver disease [13]. Fleming et al. [13] describe the latter hepatocyte progenitor cell-associated glutamine synthetase expression pattern as aggregates of glutamine synthetase-positive hepatocytes, "buds," seen mostly in areas of parenchymal extinction, and thought to be arising from/budding off of stem cells located in ducts. These patterns of glutamine synthetase expression are distinct from the perivenular glutamine synthetase reactivity juxtaposed to portal tracts that typifies regressed cirrhosis. Finally, in our normal liver cases, no glutamine synthetase immunoreactivity was observed around portal tracts; rather, these cases exhibited the expected normal pattern for glutamine synthetase around central veins.

While our study included cases encompassing a variety of etiologies for advanced liver disease, primary biliary cholangitis, and primary sclerosing cholangitis were excluded because the pattern of fibrosis in these disorders differs from other common causes of cirrhosis due to hepatitic injury. In contrast to the pattern of parenchymal extinction, fibrosis, and juxtaposition of central and portal elements assessed in our study, these entities are associated with porto-central relationships that are for the most part maintained [7].

Advancements in medical therapies have introduced an era in the pathology of advanced stage hepatic disease, where a post-cirrhotic stage of liver injury is likely to become increasingly common. This new age has been heralded by the introduction of effective eradication therapy for hepatitis $\mathrm{C}$, a setting in which the regression of cirrhosis may define the morphology of liver biopsies in ongoing disease management and in explanted livers for patients still requiring a liver allograft. With the latter in mind, we anticipate that regression of cirrhosis may become more prevalent in selected hepatectomy specimens, and the absence of cirrhosis will undoubtedly be a source of confusion. It is thus increasingly important for pathologists to be able to anticipate and recognize the salient histologic features of regression. While we might reasonably expect that we will improve our skills in detecting these features over time, our ability in the short term to augment those skills with reproducible markers of regression will benefit not only the practice of pathology, but also the care of our patients. We believe that our demonstration of a characteristic immunohistochemical pattern in regression-the presence of immunoreactivity for glutamine synthetase adjacent to portal structures as a surrogate marker for venoportal approximation, is a potential bridge to guide us to a more confident diagnosis of regression of cirrhosis in current medical practice.
Acknowledgements We thank Dr. Ian Wanless for his guidance and input with this work, as well as Dr. Charlie Alpers (Professor and Interim Chair, University of Washington Department of Pathology), Dr. David Chhieng (Director of Anatomic Pathology, University of Washington) and the Anatomic Pathology Department at the University of Washington for providing support. We thank Bernadine Jocson (Histology Laboratory Manager, University of Washington) and the Laboratory of Immunohistochemistry (University of Washington) for assistance. This work was supported by the Human Subjects Research Division, Institutional Review Board (IRB) (IRB\#52314).

Funding There are no significant relationships with, or financial interest in, any commercial companies pertaining to this article.

\section{Compliance with ethical standards}

Conflict of interest The authors declare that they have no conflict of interest.

Publisher's note: Springer Nature remains neutral with regard to jurisdictional claims in published maps and institutional affiliations.

\section{References}

1. Scaglione S, Kliethermes S, Cao G, et al. The epidemiology of cirrhosis in the United States: a population-based study. J Clin Gastroenterol. 2015;49:690-696.

2. Williams R. Global challenges in liver disease. Hepatology. 2006;44:521-526.

3. Heidelbaugh JJ, Bruderly M. Cirrhosis and chronic liver failure: part I. Diagnosis and evaluation. Am Fam Physician. 2006;74:756-762.

4. MacSween RNM, Burt AD, Portmann B, et al. Structure, function, and responses to injury. In: MacSween's pathology of the liver. Edinburgh, New York: Churchill Livingstone/Elsevier; 2012. p. $1-87$.

5. Stueck AE, Wanless IR. Hepatocyte buds derived from progenitor cells repopulate regions of parenchymal extinction in human cirrhosis. Hepatology. 2015;61:1696-1707.

6. Wanless IR, Nakashima E, Sherman M. Regression of human cirrhosis. Morphologic features and the genesis of incomplete septal cirrhosis. Arch Pathol Lab Med. 2000;124:1599-1607.

7. Quaglia A, Alves VA, Balabaud C, et al. Role of aetiology in the progression, regression, and parenchymal remodelling of liver disease: implications for liver biopsy interpretation. Histopathology. 2016;68:953-967.

8. Sun Y, Zhou J, Wang L, et al. New classification of liver biopsy assessment for fibrosis in chronic hepatitis B patients before and after treatment. Hepatol Balt Md. 2017;65:1438-1450.

9. McGovern BH, Abu Dayyeh BK, Chung RT. Avoiding therapeutic pitfalls: the rational use of specifically targeted agents against hepatitis C infection. Hepatology. 2008;48:1700-1712.

10. Iredale JP. Models of liver fibrosis: exploring the dynamic nature of inflammation and repair in a solid organ. J Clin Invest. 2007;117:539-548.

11. Iredale JP, Benyon RC, Pickering J, et al. Mechanisms of spontaneous resolution of rat liver fibrosis. Hepatic stellate cell apoptosis and reduced hepatic expression of metalloproteinase inhibitors. J Clin Invest. 1998;102:538-549.

12. Marcellin P, Gane E, Buti M, et al. Regression of cirrhosis during treatment with tenofovir disoproxil fumarate for chronic 
hepatitis B: a 5-year open-label follow-up study. Lancet. 2013; 381:468-475.

13. Fleming KE, Wanless IR. Glutamine synthetase expression in activated hepatocyte progenitor cells and loss of hepatocellular expression in congestion and cirrhosis. Liver Int. 2013;33:525-534.

14. Kuo FC, Darnell JE. Evidence that interaction of hepatocytes with the collecting (hepatic) veins triggers position-specific transcription of the glutamine synthetase and ornithine aminotransferase genes in the mouse liver. Mol Cell Biol. 1991; 11:6050-6058.

15. Racine-Samson L, Scoazec J, D'Errico A, et al. The metabolic organization of the adult human liver: a comparative study of normal, fibrotic, and cirrhotic liver tissue. Hepatology. 1996;24: 104-113. 